\title{
Measuring of plasma's velocity in electrodynamic railgun using high-frequency method
}

\author{
Vladimir $V$. Kirievskiy, and Evgeny $V$. Kirievskiy* \\ Platov South-Russian State Politechnic University (NPI), 346400 Novocherkassk, Russian Federation
}

\begin{abstract}
A method is proposed for measuring the velocity of a moving plasma in an electrodynamic railgun in the part of internal ballistics. The achieved result is to provide a measurement of instantaneous velocity and an increase of the safety level for operation of measuring devices.

The method is based on the excitation of the rail system of railgun high frequency electric oscillations during in due motion of plasma and creates current resonance. Then is continuously measured cyclic resonance frequency, and the instantaneous velocity of the plasma is determined by a formula, that consist of the current time, the cyclic resonance frequency, equivalent inductance of the power supply of railgun, linear inductance and rail length, and as well used as a model of measurement measure capacitance of capacitor, connected in parallel to rail of railgun grounded from one side. Application of plasma velocity measuring method is suitable for the highly-accurate control of plasma acceleration and pushed it to the railgun bodies with controlled acceleration, requiring measurement during movement of the plasma instantaneous values of velocity.
\end{abstract}

\section{Introducing}

There are a number of applications of a special class of electrophysical equipments, called "Electrodynamic Magnetoplasma Accelerator" (EMA) or else the "railgun" [1-7]. Most of these applications at the stage of experimental studies require the measurement of the average velocity at the base interval path, which does not pose any difficulties, because they use a simple so-called "time-of-flight method" of measurement. The essence of this method is to measure the average velocity as the ratio of the base path interval between a pair of neighboring induction sensors to the time of passage by the plasma clot of this path interval.

However, there is a special application of EMA, it is necessary to measure not the average but instantaneous, or at least quasi-instantaneous velocity of the plasma in railgun on the area of the internal ballistics. Such a problem arises, for example, if it is necessary to include into the control system of the EMA a correction circuit of the plasma velocity in the area of internal ballistics to provide a given output velocity in the EMA.

\footnotetext{
* Corresponding author ekir99@mail.ru
} 


\section{High-frequency method of measurement}

To solve this problem, for example, can be used a way of measuring the velocity of moving plasma in EMA [7, 8], based on excitation in a rail system of EMA high-frequency electric oscillations during the motion of the plasma in the area of the internal ballistics. In this case the oscillation frequency is set fixed, and the velocity of the plasma is defined as the quotient of half the wavelength of the excited electric oscillations on the time interval between the neighboring amplitudes bursts of the excited electric oscillations.

As follows from the above method [1-7], it also applies the time-of-flight measurement method, but as the base distance is not used the path interval between neighboring induction sensors on the trajectory of motion, but half the wavelength of excited electrical oscillations, which is thousands of times less than the path interval between neighboring induction sensors. Thus, it can be argued that such a drastic reduction in the base path interval leads to the measurement of the quasi-instantaneous plasma velocity in the EMA .

The disadvantages of the described method [9] include the need for physical connection of the output of the measuring device simultaneously to two rails of the EMA, the potential difference between which in the process of operation of the accelerator reaches 100-200 $\mathrm{kV}$, and as a consequence - there is an increased risk of its failure and damage to the operator by electric current, as well as the inability to measure instantaneous speed, which would improve the accuracy of measuring the plasma movement and control of the EMA.

\section{Theoretical basis of the resonance method for velocity's measuring}

The technical result of the solution proposed in this article is to provide instantaneous velocity measurement and increase the level of safety of the measuring devices implemented on its basis. The task is achieved by the fact that in the above method of measuring the velocity of the moving plasma in the EMA [10], based on the excitation in the rail system of high-frequency electrical oscillations during the movement of the plasma, on the site of the internal ballistics in the rail system of the accelerator create a current resonance, continuously measure the cyclic resonance frequency $\omega(t)$, and the plasma velocity is determined by the formula:

$$
v(t)=\frac{d}{d t}\left[\frac{\psi(t)+\sqrt{\psi(t) \cdot(\psi(t)+L)}}{b}\right],
$$

where: $t$ - time, $\psi(t)=b \cdot l-\frac{1}{\omega(t)^{2} C}, L-$ equivalent inductance of the power supply for the accelerator, $b$ - linear inductance of the rail, $l$ - rail length, $C$ - used as a model measure of measuring the capacitance of the capacitor connected in parallel to the grounded one end of the rail [18].

The proposed technical solution is illustrated in Fig. 1-3.

For Fig. 1 in a simplified form shows the structure of the EMA powered by a capacitor bank and its equivalent electrical circuit. The EMA consists of a power supply 1, a plasma clot (armature) 2, an accelerated body 3 and a rails 4, one of which (lower in Fig. 1) is grounded. In the wiring diagram: $C 5$ and $C 6$ - respectively equivalent capacity and inductance of the power supply, $L 7$ and $L 8$ - components of the distributed inductance of the rails until the current position of the plasma armature (PA), $R 9$ - equivalent electrical resistance of the PA, $L 10$ and $L 11$ - components of the distributed inductance of the rails 
after the current position of the PA. The points 12 and 13 of the electrical circuit correspond to the input ends of the rails (the place of the beginning of the PA), 14 and 15 - to the current position of the PA, 16 and 17 - to the output ends of the rails (the place of the PA exit from the channel of EMA).

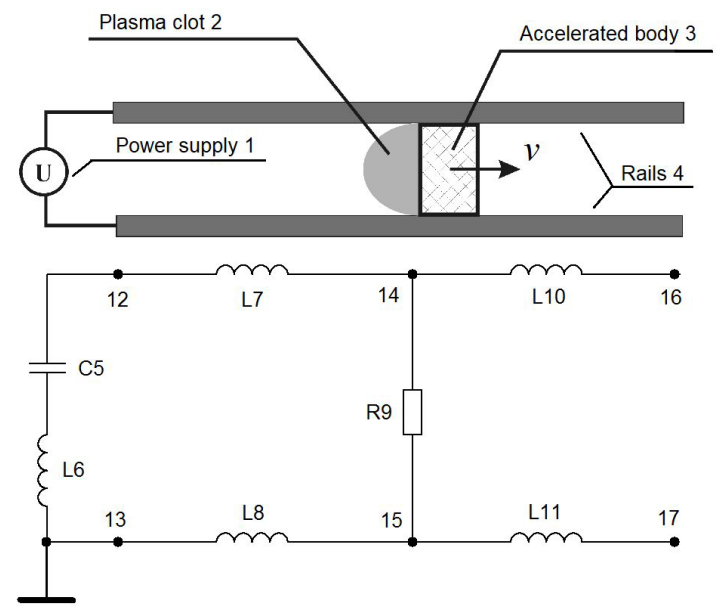

Fig. 1. Structure of the EMA powered by a capacitor bank and its equivalent electrical circuit.

It is obvious that the parameters $L 7, L 8, L 10$ and $L 11$ are linear functions of the PA coordinate $x(t)$ and in the case of an identical geometric design of the rails and the uniform distribution of the linear inductance $b$ will be observed $L 7=L 8 ; L 10=L 11$. For example, typical values for rails of length $l=1 \mathrm{~m}$ are $L 7+L 8+L 10+L 11=0,12 \mu \mathrm{H}$, which corresponds to the linear inductance of the rails $b=0,06 \mu \mathrm{H} / \mathrm{m}$. The values of the parameters $C 5$ and $L 6$ are determined exclusively by the electromechanical design of the EMA (typical values are the $C 5 \approx 400 \mu \mathrm{F}$ and $L 6 \approx 0,2 \mu \mathrm{H}$ ) and remain unchanged throughout the acceleration period. The value of the parameter $R 9$ in the acceleration process varies in accordance with the variations in the width of the PA (typical value $R 9 \approx 0,1 \mathrm{Ohms}$ ), but the amplitude of its changes is insignificant and does not significantly affect the frequency characteristics of the electrical circuit shown in Fig.1.

The resonant cyclic frequency $\omega(t)$ of the oscillatory circuit formed by the elements , $C 5, L 6, L 7, L 8$ and $R 9$ in the process of motion of the PA is a function of the PA's coordinate ${ }^{x(t)}$ and is determined by the ratio:

$$
\omega(t)=\frac{1}{\sqrt{(2 b x(t)+L 6) \cdot C 5}} .
$$

For the above typical circuit parameters, the frequency range is $14.0-17.8 \mathrm{kHz}$. Therefore, for the total acceleration time (typical value of about $400 \mu \mathrm{s}$ ), the possible number of frequency measurements (and hence the velocity of PA) will be on average no more than 12 (by the number of full half-cycles of current/voltage, within the total acceleration time), which is very small, as it can provide a measurement of not instantaneous (or, more precisely, quasi-instantaneous), but only the average velocity of PA on a very small number of parts of the internal trajectory.

To move to the measurement of instantaneous velocity, it is necessary to significantly increase the resonance frequency of the oscillating circuit, which includes areas of variable inductance, linearly dependent on the coordinates of the PA. To do this in the diagram between points 13 and 17 in Fig. 1 (i.e. parallel to the EMA grounded rail, which is 
advisable from the point of view of the plant operation safety), it is proposed to include an additional small known capacity in the form of a model capacitor (about several hundre $\mathrm{pF}$ ).

In Fig. 2 shows the equivalent electrical circuit of the power part of the EMA, illustrating the proposed method of velocity measurement. Compared to the diagram shown in Fig. 1, it additionally contains the capacitance of the sample capacitor $C 18$ and the generator 19. The capacitance of the sample capacitor C18 is included in the frequency-setting circuit of the generator.

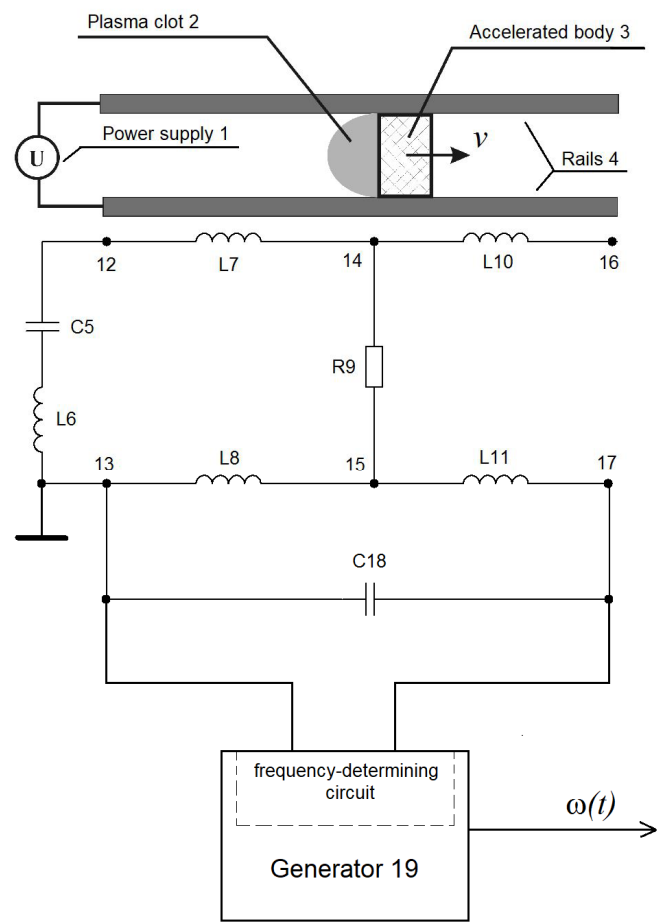

Fig.2. Equivalent electrical circuit of the power part of the EMA.

Exciting the oscillating circuit formed by the elements $C 18, C 5, L 6, L 7, L 8, L 11$ and $R 9$ at the upper resonant frequency:

$$
\omega(t)=\frac{1}{\sqrt{L_{e q} C 18}},
$$

where $L_{e q}=b l+b x(t) \cdot\left(\frac{L 6+b x(t)}{L 6+2 b x(t)}-1\right), \quad$ use the frequency $\omega(t)$ as an informative parameter to determine the coordinates $x(t)$ of the PA by the formula obtained from the formula (2):

$$
x(t)=\frac{\psi(t)+\sqrt{\psi(t) \cdot(\psi(t)+L 6)}}{b},
$$

$$
\text { where } \psi(t)=b \cdot l-\frac{1}{\omega(t)^{2} C 18 .}
$$


The motion's velocity of PA is determined by the formula (3) using differentiation (2) as a derivative of the time from the coordinate ${ }^{x(t)}$ of PA:

$$
v(t)=\frac{d}{d t}\left[\frac{\psi(t)+\sqrt{\psi(t) \cdot(\psi(t)+L 6)}}{b}\right]
$$

By entering the notation $C \equiv C 18$ and $L \equiv L 6$, finally get:

$$
v(t)=\frac{d}{d t}\left[\frac{\psi(t)+\sqrt{\psi(t) \cdot(\psi(t)+L)}}{b}\right],
$$

where $\psi(t)=b \cdot l-\frac{1}{\omega(t)^{2} C}$.

Curve 20 in Fig3 shows the dependence of the measured frequency on the coordinate $x(t)$ for the above typical values of the circuit parameters.

The dependence of the measured frequency on the coordinate $x(t)$, as seen from the graph in Fig. 3, has the character close to linear, which simplifies the construction of devices that implement the described method of measurement.

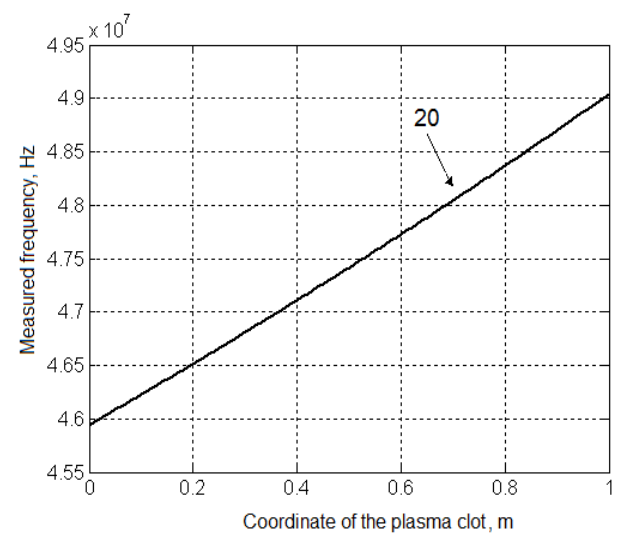

Fig.3. Graf of the dependence "measured frequency on the coordinate $x(t)$ ".

The range of the measured frequency is $46-49 \mathrm{MHz}$, which will allow to carry out in the process of acceleration up to 19 thousand samples of the PA coordinate (by the number of full half-cycles of current/voltage, within the total acceleration time) and therefore to provide measurement of almost instantaneous velocity at any point of the internal ballistics trajectory.

\section{Conclusion}

Thus, the proposed method [10] allows to measure the instantaneous velocity of the moving plasma in the EMA and to increase the level of safety of the measuring devices implemented on its basis.

It is most advisable to use it in electrophysics for high-precision control of the acceleration of the plasma and the bodies pushed by it in the EMA with controlled acceleration, requiring measurement during the movement of the plasma, not average, but instantaneous values of velocity to control the acceleration process according to the results of continuous velocity measurement. 


\section{References}

1. B. Azanov, V. A. Alexandrov, S. S. Obydennikov, IEEE Trans. Magn., 33, 1, 213 (1997)

2. V. Kozlov, S. N. Luzganov, V. V. Polistchook, A. V. Shurupov, IEEE Trans. Magn., 1, 146 (2004)

3. V. V. Viktorov, T. M. Karadzhali, A.S. Chursin, Instruments and Experimental Techniques, 5, 212 (1983)

4. K. E. Nalty, R. C. Zowarka, L. D. Holland, IEEE Trans. Magn., 20, 2, 328 (1984)

5. V. A. Viktorov, B.B. Lunkin, A.S. Sovlukov, Vysokochastotnyy metod izmereniya neelektricheskikh velichin (Nauka, Moscow, 1978)

6. V. A. Viktorov, B.V. Lunkin, A.S. Sovlukov, Radiovolnovyye izmereniya parametrov tekhnologicheskikh protsessov (Energoatomizdat, Moscow, 1989)

7. Ye. V. Kiriyevskiy, Obzor metodov i sredstv izmereniya skorosti razgona tel $v$ magnitoplazmennom uskoritele mass (Yuzh.-Ros. gos. tekhn. un-t., Novocherkassk, 2003) Dep. in VINITI 18.02.2003, №321-B2003.

8. Ye.V. Kiriyevskiy, Izmereniye parametrov dvizheniya tel $v$ plazmennykh elektrodinamicheskikh uskoritelyakh (Parametricheskiy i strukturnyy sintez izmeritel'nykh preobrazovateley) (SKNTS VSH, Rostov-na-Donu, 2005)

9. M.L. Sloan, IEEE Trans. Magn., 22, 06, 1746 (1986)

10. V.E. Kirievsky, E.V. Kirievsky, Patent RU 2651633 (12.09.2016) 